\title{
Describing Droplet Motion on Surface-Textured Ratchet Tracks with an Inverted Double Pendulum Model
}

Mayssam Naji, Ecem Yelekli Kirici, Ali Javili, and E. Yegan Erdem*

Cite This: Langmuir 2021, 37, 4810-4816

Read Online

ACCESS | Llll Metrics \& More | 回 Article Recommendations

\begin{abstract}
We describe the motion of a droplet on a textured ratchet track using a nonlinear resonator model. A textured ratchet track is composed of a semicircular pillar array that induces a net surface tension local gradient on a droplet placed on it. When a vertical vibration is applied, hysteresis is overcome, and the droplet moves toward the local lower energy barrier; however, due to the repetitive structure of texture, it keeps moving until the end of the track. The droplet motion depends on the amplitude and frequency of the vertical oscillation, and this dependence is nonlinear. Therefore, finding a fully analytic solution to represent this motion is not trivial. Consequently, the droplet motion remains poorly understood. In this study, we elaborate on the utility of a double pendulum as a basis for modeling the droplet motion on surfaces inducing asymmetric force. Similar to the droplet motion, resonators, such as a double pendulum, are simple, yet nonlinear systems.

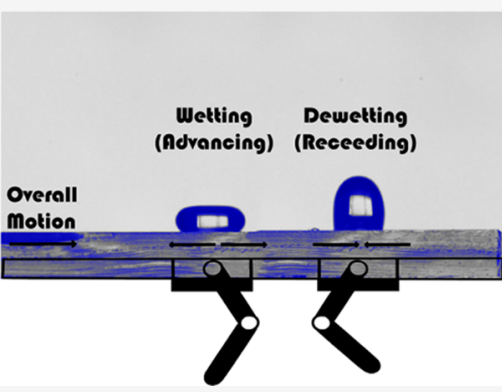
Moreover, an inverted double pendulum motion has key characteristics such as the two-phase motion and the double peak motion, which are also observed in the droplet motion. We use various data-processing methods to highlight the similarity between these two systems both qualitatively and quantitatively. After establishing this comparison, we propose a model that utilizes an inverted double pendulum mounted on a moving cart to successfully simulate the motion of a droplet on a ratchet track. This methodology will lead to the development of an accurate droplet-motion modeling approach, and we believe that it will be useful to understand droplet dynamics more deeply.
\end{abstract}

\section{INTRODUCTION}

Recent developments in the field of microfluidics have brought fully integrated devices for biomedical and chemical applications. $^{1-8}$ This progress has heavily relied on understanding droplet manipulation techniques. ${ }^{9-12}$ Droplet-based flow provides benefits such as elimination of cross contamination, less material consumption, better concentration control, and rapid mixing. ${ }^{13}$ On the other hand, manipulation of droplets on surfaces rather than in channels brings extra benefits including elimination of pumps, leakages, and clogging and addressing samples individually. ${ }^{14}$ One way of achieving droplet motion on surfaces is utilizing an electric field, known as electrowetting, ${ }^{15-19}$ and other methods include methods that use thermocapillary forces ${ }^{20-22}$ and chemical gradients. ${ }^{23-25}$ As an alternative to these methods, droplet manipulation can also be achieved by creating local energy gradients with surface texture. $^{26-31}$ The surface can be composed of a texture pattern that enables a continuous cycle of wetting and dewetting. ${ }^{32}$ In the method introduced by Shastry et al., local surface energy gradients were generated using pillar arrays that are arranged with decreasing distance where a vertical vibration was also applied to overcome hysteresis. ${ }^{28}$ A continuous propagation was later achieved by utilizing semicircular pillar arrays that induce asymmetric surface forces on the droplet's three-phase contact line, creating a surface energy local gradient and resulting in a biased-oscillating driving force. ${ }^{29}$ Extensive work has been done on studying the relationship between this directed motion and input parameters (frequency and amplitude) and droplet properties such as volume and chemical composition. $^{28,29,31,33,34}$ On the other hand, theoretical works examining the droplet motion on a textured ratchet described by Shastry et $\mathrm{al}^{28}$ and Duncombe et al. ${ }^{29}$ have been relatively less numerous compared to other droplet-based systems. Due to the complex nature of this motion and its nonlinear behavior, an accurate theoretical reproduction of this motion is currently not available. Most theoretical works on textured surfaces have been focused on modeling droplet-surface interactions such as dropletimprint shape. ${ }^{35,36}$ Dong et al. proposed a mass-springdamper system which translated vertical vibrations into a horizontal motion. ${ }^{34}$

In this work, we compare the motion of the double pendulum on a cart to the droplet motion. Then, we introduce a novel model for understanding droplet motion on surface-textured ratchet tracks composed of semicircular pillar arrays as described

Received: December 23, 2020

Revised: April 1, 2021

Published: April 14, 2021

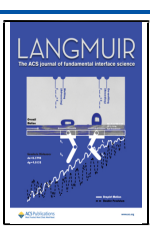


in refs 29 and 31. The studied surface is shown in Figure 1. This model is based on the motion of an inverted double pendulum

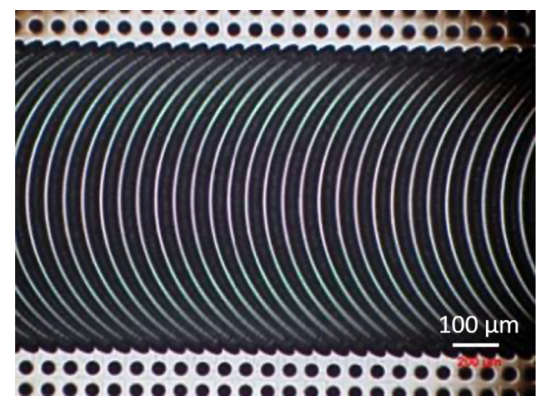

Figure 1. Top view of the ratchet track. The pattern imprinted on a PDMS substrate is composed of an array of arc-shaped pillars.

mounted on a cart. It can be seen from experimental data and from several numerical observations that both the double pendulum and droplet motion show resembling responses. Moreover, the double pendulum is a well-studied nonlinear system; ${ }^{37,38}$ therefore, it serves as the basis of the droplet motion model.

In this study, both the common elements between a double pendulum response and a droplet motion are highlighted, and an elastic functional data analysis (EFDA) ${ }^{39}$ is applied for a detailed comparison. Finally, a simple numerical model based on the motion of a double pendulum is proposed. The proposed model is then quantitatively compared to the experimental findings using the Fréchet distance, ${ }^{40}$ which is used for similarity calculations. ${ }^{41}$ The experimental data consist of videos collected with a high-speed camera of a water droplet moving on a ratchet track. Overall, this work presents a theoretical framework that can be used to simulate a droplet motion on a surface-textured ratchet track.

\section{DATA COLLECTION AND PROCESSING}

Droplets are transported in the "Fakir" state on a textured surface ratchet track. ${ }^{29}$ The track is composed of an array of arcshaped pillars, and therefore, the leading edge of the droplet consistently experiences a stronger pining force. The track is subjected to sinusoidal vertical vibration which alternatively sets the droplet in advancing and receding modes where dewetting and wetting of the surface occur in a sequence while eliminating the pinning due to hysteresis. This sets the droplet in an overall horizontal motion.

When the tracks oscillate vertically, it puts the droplet contact line in cyclic oscillation. The droplet oscillation is maximized when it is excited at the resonance frequency. However, unless the signal amplitude is above a threshold value, the droplet will remain pinned to the track. When the hysteresis is overcome with vertical vibration, even though the droplet contact line goes through the cycle of expansion and recession, it experiences a net forward force in the horizontal direction. ${ }^{28,29}$

In this study, water droplets of typical volumes between 5 and $10 \mu \mathrm{L}$ are experimented on the ratchet track. The track is placed on a stage mounted on top of a vibration actuator. A signal generator and an amplifier set the actuator to oscillate with a predetermined frequency in the range of $45-85 \mathrm{~Hz}$ and an amplitude ranging from $2 \mathrm{G}$ to $10 \mathrm{G}$ (Figure 2). At each frequency, a video of the silhouette of the droplet is recorded using a high-speed camera $(10,000 \mathrm{fps})$, then it is processed using image processing libraries in MATLAB. The stages of the processing and an example of the data obtained are shown in Figure 2. The experimental setup consisted of a vibration actuator (Brüel Kjær Type 4809), a function generator (GW Instek type SFG 2004), an amplifier (Bruel Kjaer-type 2718), an oscilloscope (DSOX2012A), an accelerometer (Dytran 3035B1), a high-speed camera (Phantom MIRO LC110), and a high intensity light source (VRI-MICROBEAM-128).

\section{MODELING}

The motion of a double pendulum on a cart has been wellstudied to date. ${ }^{42,43}$ Although there are several types of resonator models available, a double pendulum is a relatively simple, yet highly nonlinear system. ${ }^{44}$ Moreover, the double pendulum simulated motion and droplet recorded motion share similar attributes as highlighted in Figure 3. Hence, the double pendulum on a cart can be used to understand and re-create the behavior of the droplet on the ratchet track. The theoretical framework consists of two links with a uniform density of 2700 $\mathrm{kg} / \mathrm{m}^{3}$ forming a double pendulum attached to a moving cart on a horizontal track. The cart does not experience any friction when sliding on the track. The cart and pendulum joints are modeled as massless objects with no damping coefficients and no external power applied on them. MATLAB's Simscape tool is used to produce a stable simulation of the double pendulum motion; the schematic of this virtual set up is shown in Figure 3. At equilibrium, the pendulum remains in the inverted position even when subjected to a random disturbance. In order to maintain this state, the pole placement technique ${ }^{45}$ is used to compute the control gains from the linearized model.

Finally, in order to set the pendulum in motion, it is modeled in an anisotropic environment where an external universal force sets the double pendulum into motion. This external bias force is selected such that it can simulate the input that the droplet on a textured ratchet track under vertical vibration experiences. The vertical oscillations given to the droplet result in the oscillation

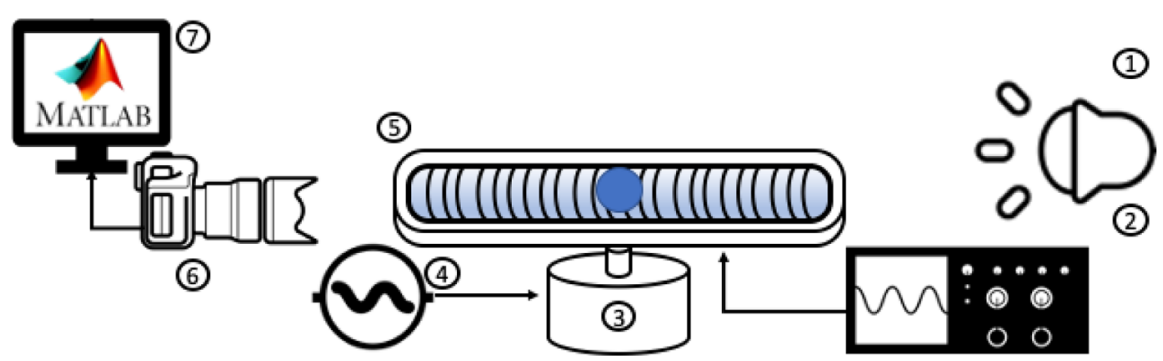

Figure 2. Experimental setup and resources used in collecting and processing data: (1) soft white LED light, (2) oscilloscope, (3) vibration actuator, (4) signal generator, (5) PDMS ratchet track, (6) high-speed camera, and (7) MATLAB script based on an image-processing tool box. 


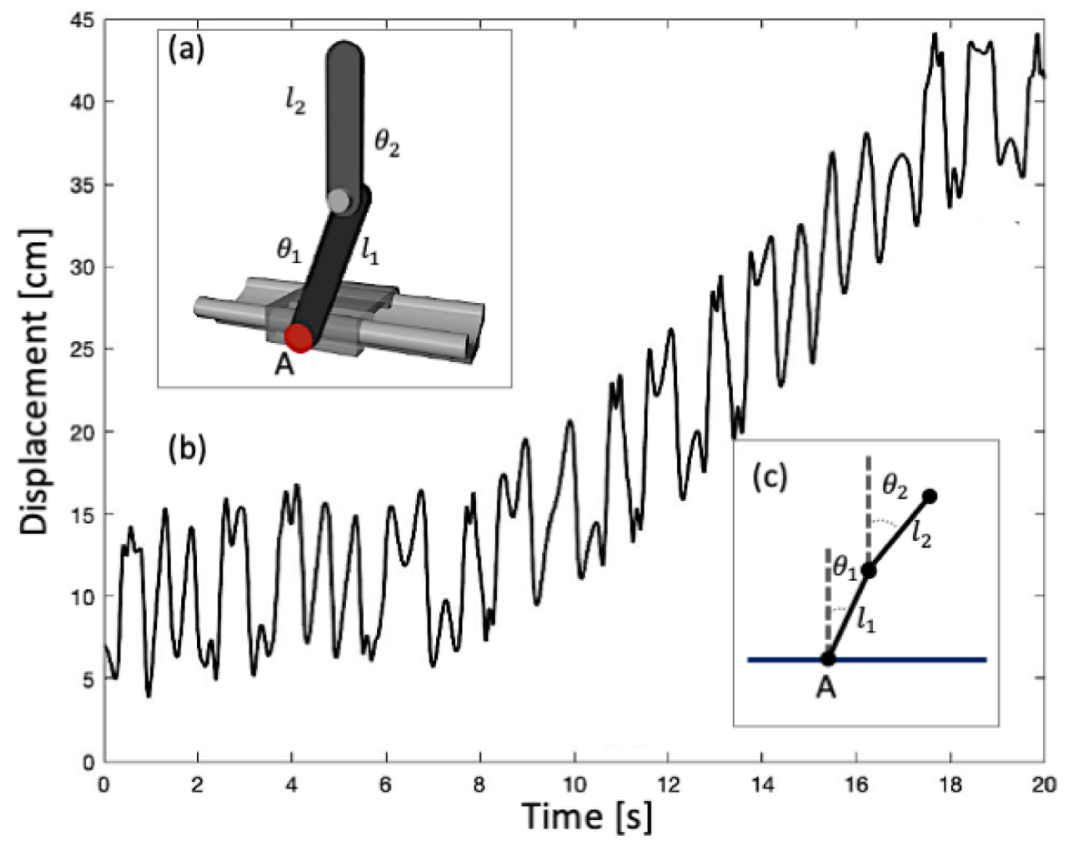

Figure 3. Typical behavior of an inverted double pendulum on a sliding cart obtained with Simscape Multibody in MATLAB. (a) Visual presentation of the simulated system. The double pendulum has dimensions $L_{1}=18 \mathrm{~cm}$ and $L_{2}=14 \mathrm{~cm}$, with a uniform density of $2700 \mathrm{~kg} / \mathrm{m}^{3}$. The initial angles are $\theta_{1}=-20^{\circ}$ and $\theta_{2}=0^{\circ}$. The double pendulum does not have any initial velocity. (b) Displacement of the pendulum according to point $\mathrm{A}$ with respect to time when an external bias force is applied. (c) Schematic showing the reference points for $L_{1}, L_{2}, \theta_{1}$, and $\theta_{2}$.

of the droplet contact line, and this motion causes the droplet to respond to the local energy gradient applied by the texture. The external bias force interprets these oscillations numerically into the double pendulum model as oscillations are given in the lateral direction. Specifically, the external bias force consists of a periodic input signal, similar to the input oscillations in the experiments, a random noise that compensates for the experimental errors and a bias component which corresponds to the driving force applied by the surface texture ratchet. A typical motion of the double pendulum can be seen in Figure 3b. The simulation is governed by a set of nonlinear, multivariable dynamic equations ${ }^{46}$ given in eq 1 , making it a volatile model that is highly sensitive to errors; thus, the parameters and initial conditions have to be set precisely. ${ }^{46}$ Moreover, the bias force's amplitude is in the order of $10^{-3} \mathrm{~N}$. It is observed that higher values overwrite the double peak feature while lower values do not set the pendulum into motion. In Figure $3 \mathrm{a}$, these parameters and initial conditions are presented. Similar to the droplet, there are two phases of motion in the simulation: when the pendulum experiences external bias, it oscillates around its center of mass for a certain amount of time before it moves forward.

$$
\begin{array}{ll}
a \ddot{x}+b \cos \theta_{1} \ddot{\theta}_{1}+c \cos \theta_{2} \ddot{\theta}_{2}-b \sin \theta_{1} \dot{\theta}_{1}^{2}-c & =F \\
\quad \sin \theta_{2} \dot{\theta}_{2}^{2} & \\
b \cos \theta_{1} \ddot{x}+d \ddot{\theta}_{1}+e \cos \theta_{21} \ddot{\theta}_{2}-e \sin \theta_{21} \ddot{\theta}_{2}^{2}-b g=0 \\
\quad \sin \theta_{1} & \\
c \cos \theta_{2} \ddot{x}+e \cos \theta_{21} \ddot{\theta}_{1}+f \ddot{\theta}_{2}+e \ddot{\theta}_{1}^{2} \sin \theta_{21}-c g=0 \\
\quad \sin \theta_{2}
\end{array}
$$

where $\theta_{21}=\theta_{2}-\theta_{1}$ and

$$
\begin{array}{rlrl}
a= & m_{0}+m_{1} \quad b=\frac{1}{2} m_{1} L_{1}+m_{2} L_{1} \quad c=\frac{1}{2} m_{2} L_{2} \\
& +m_{2} & \\
d= & I_{1}+\frac{1}{4} m_{1} L_{1}^{2} \quad e=\frac{1}{2} m_{2} L_{1} L_{2} \quad f=I_{2}+m_{2} L_{2}^{2} \\
& +m_{2} L_{1}^{2} & &
\end{array}
$$

The simulation parameters are the mass of the cart $\left(m_{0}\right)$, the masses of the lower and upper pendulum arms $\left(m_{1}, m_{2}\right)$, and the length of these arms $\left(L_{1}, L_{2}\right)$, and finally, the monitored output of the simulation is the position of the cart $(x)$.

The motion of the pendulum on the track exhibits two apparent modes which are also observed in the motion of a droplet on the ratchet track. These two modes directly resemble the advancing (wetting) and receding (de-wetting) state of droplet motion (Figure 4). This pattern of behavior can also be seen in the overall motion of the pendulum (Figure 3). Before overcoming hysteresis, the droplet oscillates around its center of contact line, whereas before advancing, the pendulum system oscillates around its center of mass, which shows an inherent resemblance to the droplet motion on a ratchet track. As seen in eq 2 , in the advancing state, the force at the leading edge of the droplet $\left(F_{\text {lead,adv }}\right)$ is in the same direction of the overall motion, and it exceeds the opposing force $\left(F_{\text {tail,adv }}\right)$, while in the receding (dewetting) state, the pinning force at the tail $\left(F_{\text {tail,rec }}\right)$ is in the direction of the overall motion, and it exceeds the opposing force at the leading edge $\left(F_{\text {lead,rec }}\right)$. This unbalance between the forces breaks the symmetry of the system and results in a lateral motion on the track. In parallel, the simulated pendulum has the tendency to oscillate around its center of gravity due to the fact that the simulated track induces no friction. Hence, by introducing the bias force $(F)$, this symmetry is broken and the pendulum propagation is induced as shown in eq 2 . The resultant pattern of motion will be compared to that of the droplet motion. 


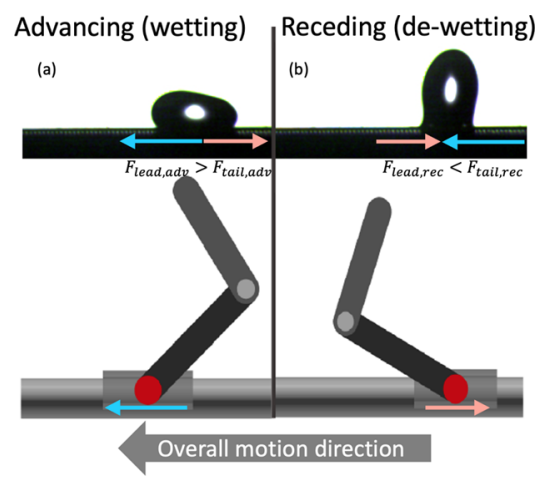

Figure 4. Droplet and pendulum systems in both advancing and receding states. (a) When a droplet is in an advancing stage, it experiences less resistance in the direction of the motion, due to the texture on the track. This scenario is similar to the motion of the pendulum when sliding in the direction of the bias. (b) On the other hand, when the droplet is in the receding stage, the leading edge of the droplet will resist the displacement, whereas the tail edge will move in the direction of the motion. Equivalently, when the pendulum moves in the opposite direction of the motion, it encounters resistance by the bias force added to the numerical model.

$$
\begin{aligned}
& \text { Advancing (wetting): } F_{\text {lead,adv }}-F_{\text {tail,adv }}>0 \\
& \text { Receding (de-wetting): } F_{\text {lead,rec }}-F_{\text {tail,rec }}<0
\end{aligned}
$$

The experimental data and the simulation results operate in different frequency domains as well as in different time and space scales (Figure 5). Hence, in order to have a meaningful comparison between the two data, the dimensions of the systems should be normalized. The data are normalized using the $Z$ Score normalization technique ${ }^{47}$ by calculating the vector-wise $Z$-score of the input signal with a center 0 and a standard deviation 1 . The assessment will solely focus on examining the motion pattern the droplet generates while moving on a ratchet track. As shown in Figure 5, the two systems exhibit similar features. In order to compare the two data, $\mathrm{EFDA}^{39}$ was utilized. EFDA models the droplet motion as a multidimensional function which enables data alignment with minimal loss. This alignment is used to calculate the geodesic distance between the two signals and to identify the significant similarity between the experimental data and the model. The geodesic distance, the shortest path length, has proven to be a useful metric for a great variety of applications in computer vision, signal, and shape analysis. ${ }^{48,49}$ Therefore, as shown in Figure 5, we align and compute the distance between the droplet and the pendulum motion for both the phase difference, $\mathrm{d} x$, and the amplitude of signal difference, $\mathrm{d} y$.

\section{METHODOLOGY}

By analyzing the experimental data, the droplet motion is identified to be composed of two major components: oscillating signal and driving signal. This observation is supported with principal component analysis (PCA), where the first two principal components recover $84 \%$ of the information (Figure 6).

The straight line component serves as the driving force that sets the droplet into forward motion. The second component encapsulates the horizontal periodic response of the droplet to the vertical oscillation of the track. This observation combined with the numerical solution of the double pendulum on a cart is the basis of our modeling approach.

$$
F(t)=V t+\phi+\alpha S
$$

Equation 3 represents the model of our system, where $S$ is the normalized signal coming from the oscillations of the double pendulum before its propagation, while $V$ is the velocity of the droplet motion; $\phi$ is the phase difference that marks the starting time of the simulation; this is used to synchronize the model results with the droplet by ensuring that both start from the same point. Moreover, $\alpha$ is the parameter that controls the nonlinearity of this model more precisely; eq 3 superimposes the driving signal, $V t+\phi$, and the pendulum oscillations (shown in Figure $6 \mathrm{~d}, \mathrm{e}$ ) according to a parameter $\alpha$. If $\alpha=0$, the result of the model will be a line, while an incremental increase in the value of $\alpha$ will gradually increase the presence of $S$ in the final generated signal. The final outcome of eq 3 is $F(t)$, which is the model result of the input, the droplet motion. The parameter $\alpha$ plays an important role in tailoring the model to the experiment. Using the Fréchet distance, ${ }^{50}$ shown in eq 4, $\alpha$ can be varied to maximize the similarity between the model and the experiment.

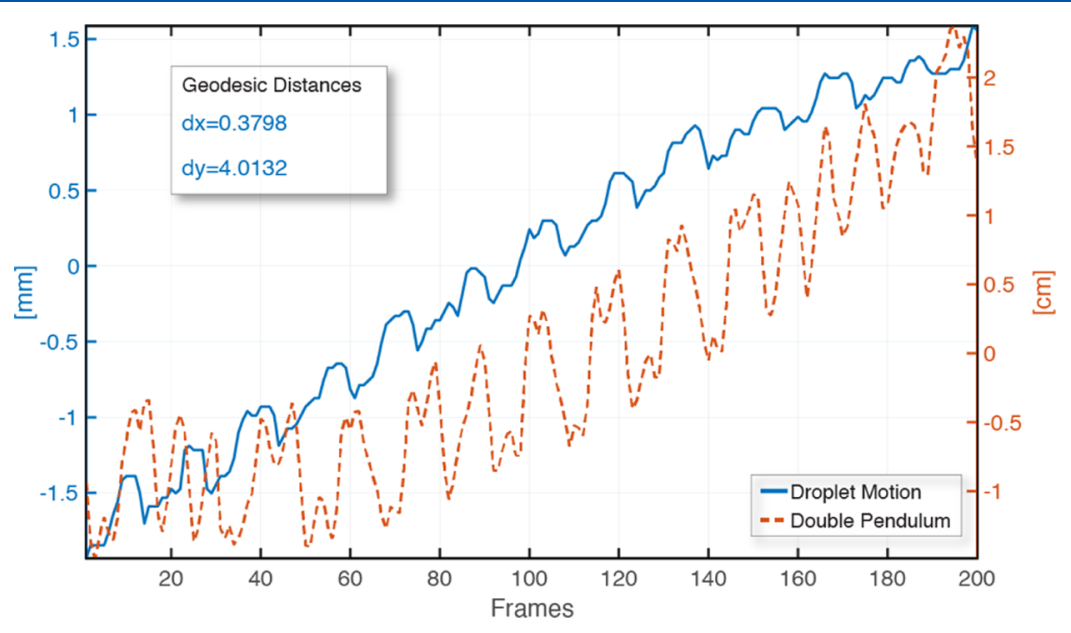

Figure 5. In blue is the droplet motion of a water droplet on a track oscillating with a frequency of $60 \mathrm{~Hz}$. In orange is the motion of an actuated double pendulum of dimensions $L_{1}=18 \mathrm{~cm}$ and $L_{2}=14 \mathrm{~cm}$, with a uniform density of $2700 \mathrm{~kg} / \mathrm{m}^{3}$ and initial angles $\theta_{1}=0$ and $\theta_{2}=-20^{\circ}$. Both functions are normalized, then the elastic geodesic distance is calculated ( $\mathrm{d} y$ : amplitude distance, $\mathrm{d} x$ : phase distance). 
(a)

\begin{tabular}{|c|c|c|c|c|}
\hline Principal Component & $1^{\text {st }}$ & $2^{\text {nd }}$ & $3^{\text {rd }}$ & others \\
\hline Variance (\%) & 75.85 & 8.49 & 6.04 & 9.62 \\
\hline Cumulative Variance $(\%)$ & 75.85 & 84.34 & 90.38 & 100 \\
\hline
\end{tabular}

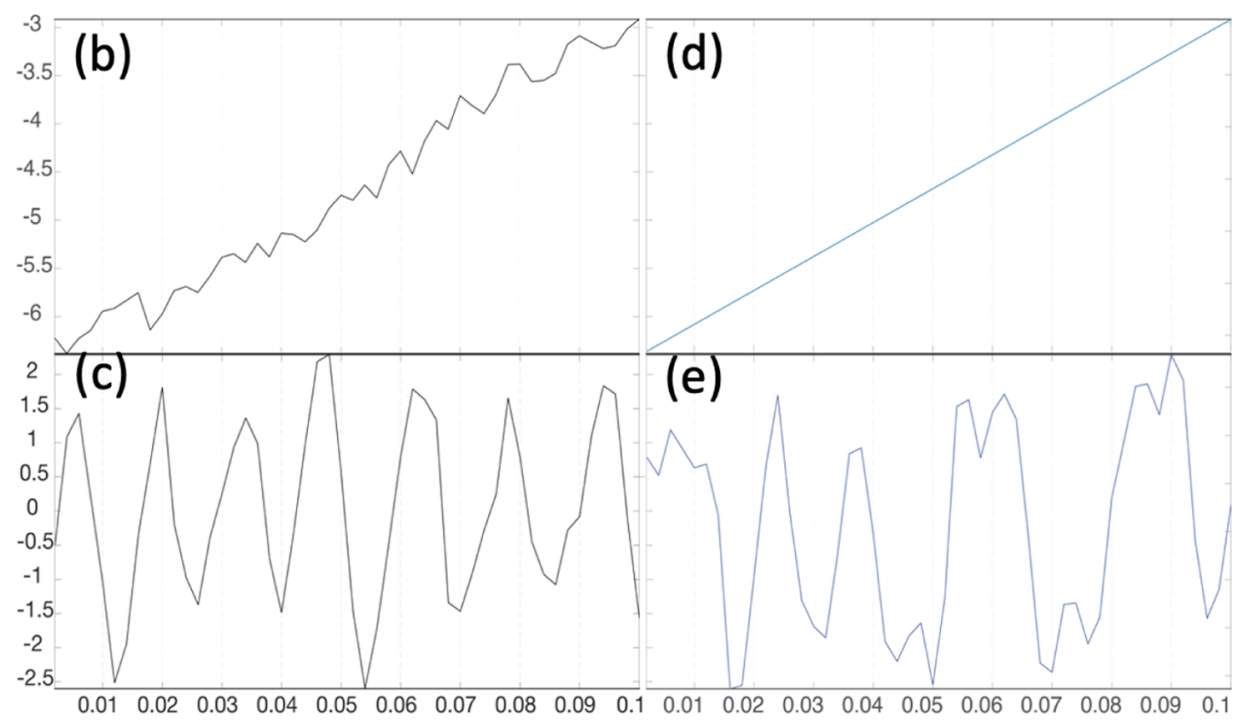

Figure 6. (a) PCA of the experimental data. It can be seen that $84 \%$ of the data can be explained by the first two principal components (PC), (b) first PC, (c) second PC, (d) first component of the model, and (e) second component of the model.

$$
\operatorname{Fr}(f, g):=\inf _{m, n} \max _{t \in[1,2]} d(f(m(t)), g(n(t)))
$$

where $d(f, g)$ is the Euclidean distance between any two trajectories such as $f$ and $g$, each parameterized in terms of $m$ and $n$.

Different weights $(\alpha)$ are used to create different models. The distance between each created model and the experiment is the optimizing factor that controls the processing analysis flow chart as demonstrated in Figure 7.

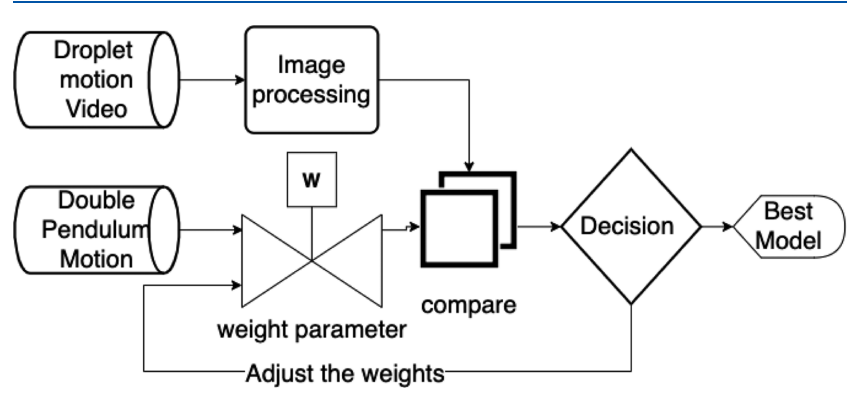

Figure 7. Flow chart explaining modeling and comparison. In the weight parameter, block $\alpha$ is changed according to the elastic distance.

\section{RESULTS}

Using the proposed pipeline in Figure 7, we can adjust the parameters in eq 3 to minimize the distance between the model output and the experimental results. After showing the similarity between a moving double pendulum and a droplet motion, we successfully re-create a signal that resembles the motion of a droplet by minimizing the Fréchet distance.

The method is tested on various experimental data. Some of the results are presented in Figure 8. In Figure 8, the leading and trailing edges are detected, and their position versus time is recorded. The midpoint between the two edges is referred to as the center of mass. The model captures the pattern of the droplet motion and reproduces different approximations for leading and trailing edges, while maintaining the difference in phase and amplitudes between the two. In Figure 8, this simple model could construct the double peak effect observed generally in droplet motion. However, when the experimental data do not exhibit this double-peak feature, it will fail to adjust to this input. Hence, it is necessary to develop a more complex system to reproduce every possible experimental output. Nevertheless, a simple superposition between a linear equation and a double pendulum simulation can re-create the general motion of a droplet while keeping its double peak feature.

\section{CONCLUSIONS}

In this paper, we have presented a simple, yet effective theoretical framework relying on the similar features of an inverted double pendulum and a droplet motion. The fact that these two models share key features such as the double peak pattern and hysteresis prior to motion gives us a deeper theoretical understanding of the droplet behavior. Both the double pendulum and droplet motion are highly complicated systems with no analytic solutions. However, the double pendulum has historically drawn significant attention, and advanced models have been developed to explain its behavior despite its complexity. In this paper, these techniques were utilized to unlock the still-uncovered droplet motion characteristics. A better theoretical understanding of droplet motion will help create more sophisticated droplet-based applications in the near future. Furthermore, theoretical research in this field does not have to start from preliminary stages. Instead, as shown in this paper, it can benefit from the other existing systems. 


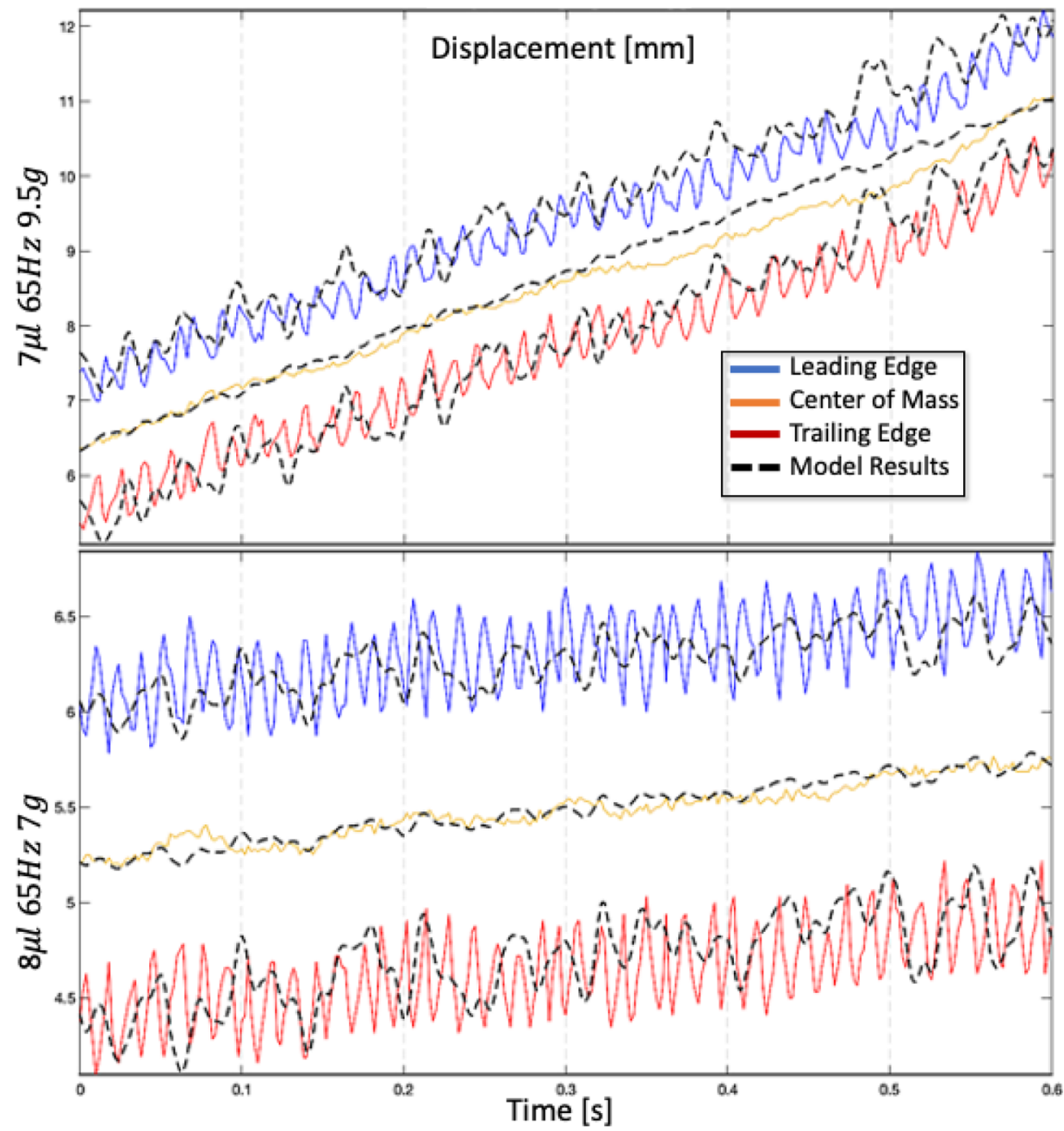

Figure 8. Comparison of the pendulum model results with the experimental data of droplets obtained for different volumes and actuation parameters.

\section{AUTHOR INFORMATION}

\section{Corresponding Author}

E. Yegan Erdem - Mechanical Engineering Department, Bilkent University, Ankara 06800, Turkey; National Nanotechnology Research Center (UNAM), Ankara 06800, Turkey;

(ㄱ) orcid.org/0000-0001-9852-2293; Phone: +90 312290

3068; Email: yeganerdem@bilkent.edu.tr

\section{Authors}

Mayssam Naji - Mechanical Engineering Department, Bilkent University, Ankara 06800, Turkey

Ecem Yelekli Kirici - National Nanotechnology Research Center (UNAM), Ankara 06800, Turkey

Ali Javili - Mechanical Engineering Department, Bilkent University, Ankara 06800, Turkey

Complete contact information is available at:

https://pubs.acs.org/10.1021/acs.langmuir.0c03610

\section{Author Contributions}

M.N. developed the model, wrote the computer codes, analyzed, and interpreted the data. E.Y.K. collected the experimental data and fabricated the ratchet tracks. A.J. helped with the interpretation of the modeling results and comparison of the two systems. E.Y.E. initiated the idea of this work, designed the model, and supervised the research. All authors contributed in the writing and reviewing of the manuscript.

\section{Notes}

The authors declare no competing financial interest.

\section{ACKNOWLEDGMENTS}

The authors thank Dr. Raji Baskaran for her support and valuable feedback on this work, Youssef Tekriti for his efforts in the design of the graphical arts, and A. Selim Çanakçı for his assistance with the experimental set-up. This work would not have been possible without the support of The Scientific and Technological Research Council of Turkey (TÜBİTAK) under the grant number 117M492.

\section{REFERENCES}

(1) Ma, Y.-D.; Luo, K.; Chang, W.-H.; Lee, G.-B. A microfluidic chip capable of generating and trapping emulsion droplets for digital loopmediated isothermal amplification analysis. Lab Chip 2018, 18, 296303.

(2) Nightingale, A. M.; Hassan, S.-u.; Evans, G. W. H.; Coleman, S. M.; Niu, X. Nitrate measurement in droplet flow: gas-mediated crosstalk and correction. Lab Chip 2018, 18, 1903-1913.

(3) Lignos, I.; Morad, V.; Shynkarenko, Y.; Bernasconi, C.; Maceiczyk, R. M.; Protesescu, L.; Bertolotti, F.; Kumar, S.; Ochsenbein, S. T.; Masciocchi, N.; Guagliardi, A.; Shih, C.-J.; Bodnarchuk, M. I.; deMello, A. J.; Kovalenko, M. V. Exploration of near-infrared-emissive colloidal multinary lead halide perovskite nanocrystals using an automated microfluidic platform. ACS Nano 2018, 12, 5504-5517. 
(4) Feng, H.; Zheng, T.; Li, M.; Wu, J.; Ji, H.; Zhang, J.; Zhao, W.; Guo, J. Droplet-based microfluidics systems in biomedical applications. Electrophoresis 2019, 40, 1580-1590.

(5) Lin, X.; Huang, X.; Urmann, K.; Xie, X.; Hoffmann, M. R. Digital Loop-Mediated Isothermal Amplification on a Commercial Membrane. ACS Sens. 2019, 4, 242-249.

(6) Karamitros, C. S.; Morvan, M.; Vigne, A.; Lim, J.; Gruner, P.; Beneyton, T.; Vrignon, J.; Baret, J.-C. Bacterial expression systems for enzymatic activity in droplet-based microfluidics. Anal. Chem. 2020, 92, $4908-4916$.

(7) Mahmud, G.; Campbell, C. J.; Bishop, K. J. M.; Komarova, Y. A.; Chaga, O.; Soh, S.; Huda, S.; Kandere-Grzybowska, K.; Grzybowski, B. A. Directing cell motions on micropatterned ratchets. Nat. Phys. 2009, $5,606-612$.

(8) Nguyen, N.-T.; Wereley, S. T.; Shaegh, S. A. M. Fundamentals and applications of microfluidics; Artech House, 2019.

(9) Shang, L.; Cheng, Y.; Zhao, Y. Emerging droplet microfluidics. Chem. Rev. 2017, 117, 7964-8040.

(10) Sohrabi, S.; Kassir, N.; Keshavarz Moraveji, M. Droplet microfluidics: fundamentals and its advanced applications. RSC Adv. 2020, 10, 27560.

(11) Teh, S.-Y.; Lin, R.; Hung, L.-H.; Lee, A. P. Droplet microfluidics. Lab Chip 2008, 8, 198-220.

(12) Atencia, J.; Beebe, D. J. Controlled microfluidic interfaces. Nature 2004, 437, 648-655.

(13) Suea-Ngam, A.; Howes, P. D.; Srisa-Art, M.; deMello, A. J. Droplet microfluidics: from proof-of-concept to real-world utility? Chem. Commun. 2019, 55, 9895-9903.

(14) Malinowski, R.; Parkin, I. P.; Volpe, G. Advances towards programmable droplet transport on solid surfaces and its applications. Chem. Soc. Rev. 2020, 49, 7879-7892.

(15) Pollack, M. G.; Shenderov, A. D.; Fair, R. B. Electrowetting-based actuation of droplets for integrated microfluidics. Lab Chip 2002, 2, 96-101.

(16) Mugele, F.; Baret, J.-C. Electrowetting: from basics to applications. J. Phys.: Condens. Matter 2005, 17, R705.

(17) Fan, S.-K.; Hsieh, T.-H.; Lin, D.-Y. General digital microfluidic platform manipulating dielectric and conductive droplets by dielectrophoresis and electrowetting. Lab Chip 2009, 9, 1236.

(18) Li, J.; Kim, C.-J. C. Current commercialization status of electrowetting-on-dielectric (EWOD) digital microfluidics. Lab Chip 2020, 20, 1705-1712.

(19) Teng, P.; Tian, D.; Fu, H.; Wang, S. Recent progress of electrowetting for droplet manipulation: from wetting to superwetting systems. Mater. Chem. Front. 2020, 4, 140-154.

(20) Karapetsas, G.; Chamakos, N. T.; Papathanasiou, A. G. Thermocapillary droplet actuation: effect of solid structure and wettability. Langmuir 2017, 33, 10838-10850.

(21) Karbalaei, A.; Kumar, R.; Cho, H. Thermocapillarity in microfluidics-A review. Micromachines 2016, 7, 13.

(22) Won, B. J.; Lee, W.; Song, S. Estimation of the thermocapillary force and its applications to precise droplet control on a microfluidic chip. Sci. Rep. 2017, 7, 3062.

(23) Rossegger, E.; Hennen, D.; Griesser, T.; Roppolo, I.; Schlögl, S. Directed motion of water droplets on multi-gradient photopolymer surfaces. Polym. Chem. 2019, 10, 1882-1893.

(24) Zhao, H.; Orejon, D.; Mackenzie-Dover, C.; Valluri, P.; Shanahan, M. E. R.; Sefiane, K. Droplet motion on contrasting striated surfaces. Appl. Phys. Lett. 2020, 116, 251604.

(25) Park, J. K.; Kim, S. Droplet manipulation on a structured shape memory polymer surface. Lab Chip 2017, 17, 1793-1801.

(26) Qi, L.; Niu, Y.; Ruck, C.; Zhao, Y. Mechanical-activated digital microfluidics with gradient surface wettability. Lab Chip 2019, 19, 223-232.

(27) Lv, C.; Chen, C.; Chuang, Y.-C.; Tseng, F.-G.; Yin, Y.; Grey, F.; Zheng, Q. Substrate Curvature Gradient Drives Rapid Droplet Motion. Phys. Rev. Lett. 2014, 113, 026101.

(28) Shastry, A.; Case, M. J.; Böhringer, K. F. Directing Droplets Using Microstructured Surfaces. Langmuir 2006, 22, 6161-6167.
(29) Duncombe, T. A.; Erdem, E. Y.; Shastry, A.; Baskaran, R.; Böhringer, K. F. Controlling Liquid Drops with Texture Ratchets. Adv. Mater. 2012, 24, 1545-1550.

(30) Caballero, D.; Voituriez, R.; Riveline, D. The cell ratchet: Interplay between efficient protrusions and adhesion determines cell motion. Cell Adhes. Migr. 2015, 9, 327-334.

(31) Baştopçu, M.; Derinöz, A. E.; Yilmaz, A. C.; Erdem, E. Y. Textured surfaces as a new platform for nanoparticle synthesis. Soft Matter 2018, 14, 4311-4316.

(32) Daniel, S.; Sircar, S.; Gliem, J.; Chaudhury, M. K. Ratcheting Motion of Liquid Drops on Gradient Surfaces. Langmuir 2004, 20, 4085-4092.

(33) Caballero, D.; Katuri, J.; Samitier, J.; Sánchez, S. Motion in microfluidic ratchets. Lab Chip 2016, 16, 4477-4481.

(34) Dong, Y.; Holmes, H. R.; Böhringer, K. F. Converting Vertical Vibration of Anisotropic Ratchet Conveyors into Horizontal Droplet Motion. Langmuir 2017, 33, 10745-10752.

(35) Tretyakov, N.; Müller, M. Directed transport of polymer drops on vibrating superhydrophobic substrates: a molecular dynamics study. Soft Matter 2014, 10, 4373-4386.

(36) Ding, H.; Zhu, X.; Gao, P.; Lu, X.-Y. Ratchet mechanism of drops climbing a vibrated oblique plate. J. Fluid Mech. 2018, 835, R1.

(37) Xu, P.; Dan, Y.; Zhang, W.; Tan, Z. Improved genetic algorithm for parameters identification of cart-double pendulum. J. Vibroengineering 2019, 21, 1587-1599.

(38) Lampart, M.; Zapoměl, J. Dynamical properties of a nonautonomous double pendulum model. Math. Methods Appl. Sci. 2018, $41,7106-7114$.

(39) Tucker, J. D.; Wu, W.; Srivastava, A. Generative models for functional data using phase and amplitude separation. Comput. Stat. Data Anal. 2013, 61, 50-66.

(40) Fan, C.; Raichel, B. Computing the Fréchet Gap Distance. Discrete Comput. Geom. 2020, DOI: 10.1007/s00454-020-00224-w.

(41) Zhang, D.; Wu, Z.; Wang, X.; Lv, C.; Zhou, M. 3D non-rigid shape similarity measure based on Fréchet distance between spectral distance distribution curve. Multimed. Tool. Appl. 2021, 80, 615-640.

(42) Dorrer, C.; Rühe, J. Advancing and Receding Motion of Droplets on Ultrahydrophobic Post Surfaces. Langmuir 2006, 22, 7652-7657.

(43) Khatoon, S.; Chaturvedi, D. K.; Hasan, N.; Istiyaque, M. Optimal control of a double inverted pendulum by linearization technique. 2017 International Conference on Multimedia, Signal Processing and Communication Technologies (IMPACT), 2017; pp 123-127.

(44) Boubaker, O. The Inverted Pendulum Benchmark in Nonlinear Control Theory: A Survey. Int. J. Adv. Rob. Syst. 2013, 10, 233.

(45) Lan, Y.; Fei, M. Design of state-feedback controller by pole placement for a coupled set of inverted pendulums. IEEE 2011 10th International Conference on Electronic Measurement Instruments, 2011; pp 69-73.

(46) Önen, Ü.; Çakan, A.; İlhan, İ. Performance comparison of optimization algorithms in LQR controller design for a nonlinear system. Turk. J. Electr. Eng. Comput. Sci. 2019, 27, 1938-1953.

(47) Encyclopedia of Biometrics; Li, S. Z., Jain, A., Eds.; Springer US: Boston, MA, 2009; pp 1134-1135.

(48) Kurtek, S.; Klassen, E.; Gore, J. C.; Zhaohua Ding, Z.; Srivastava, A. Elastic Geodesic Paths in Shape Space of Parameterized Surfaces. IEEE Trans. Pattern Anal. Mach. Intell. 2012, 34, 1717-1730.

(49) Ma, F.; Wang, P.; Luo, X. A Method for Geodesic Distance on Subdivision of Trees with Arbitrary Orders and Their Applications. IEEE Transactions on Knowledge and Data Engineering, 2020; $\mathrm{p} 1$.

(50) Aronov, B.; Har-Peled, S.; Knauer, C.; Wang, Y.; Wenk, C. 12th International Conference on Multi Media Modeling, 2006; pp 52-63. 